\title{
Waveform and spectral analysis of crackles
}

\author{
M MORI, K KINOSHITA, H MORINARI, T SHIRAISHI, S KOIKE, AND S MURAO \\ From the Second Department of Internal Medicine, University of Tokyo, Tokyo, Japan
}

\begin{abstract}
Crackles were recorded from six patients, four with tuberculosis and two with chronic bronchitis. It was observed by waveform and spectral analysis that most of the frequency components of a crackle were limited within a range of $0 \cdot 1$ to $1 \mathrm{kHz}$. Characteristically, waveforms of crackles were separable into two segments, initial "starting segments" and subsequent "decay segments." It is suggested that the former represents a shock wave caused by an abrupt opening of the airway and that the latter is a damped sinusoid caused by this shock wave exciting a resonator in the lung. It is speculated that the starting segment is determined by the pressure ratio at the site of the airway opening, and the decay segment by the resonant frequency and the quality factor of the resonator. Because transmission of a crackle is highly directional the waveforms recorded on the chest wall are modified by the positional relationship between the sound source and the microphone.
\end{abstract}

It has been widely accepted that crackles are short explosive sounds generated in the lung when the airway abruptly opens. ${ }^{1}$ The timing of this opening is known to be closely associated with a particular transpulmonary pressure. ${ }^{2}$ The purpose of this study is to investigate further the origin and characteristics of crackles by waveform and spectral analysis.

\section{Methods}

The lung sounds were recorded by placing an aircoupled dynamic-type microphone firmly on the chest wall where crackles were heard by auscultation. The output of the microphone was amplified, filtered (low frequency cut-off at 100 $\mathrm{Hz}$ ) and recorded on a FM tape recorder (TEAC R210). The frequency response of the microphone and the amplifier system was flat to within $\pm 3 \mathrm{~dB}$ in the range from $0.1 \mathrm{kHz}$ to $8 \mathrm{kHz}$. Six patients, four with tuberculosis and two with chronic bronchitis, were studied. Slowing down the playback speed to one-eighth of the originally recorded tape speed, we sampled the lung sounds by means of a memory (TDK 8810) at a sampling frequency of $2 \mathrm{kHz}$ (equivalent to $16 \mathrm{kHz}$ real time) and for the time interval of 0.5 or $1 \mathrm{~s}$ (equivalent to 62.5 or $125 \mathrm{~ms}$ real time respectively). This slowing down was necessary to isolate

Address for reprint requests: Dr $\mathbf{M}$ Mori, Second Department of Internal Medicine, University of Tokyo, Hongo, Bunkyo-ku, Tokyo, Japan. and sample a single crackle. The sampled waveforms were monitored and displayed by a jet-type recorder (Mingograf, Siemens). For the waveform analysis the time scale was expanded 1600 times, eight times by slowing down the play-back speed and 200 times by reducing the read-out frequency from the memory from $2 \mathrm{kHz}$ to $10 \mathrm{~Hz}$. For the spectral analysis (Fourier transform) the same signals stored in the memory were transferred back to the FM tape recorder, sampled by an analog-digital converter (TOSBAC T-40) and analysed by a computer (ACOS 600). This time the sampling frequency and the time window were $64 \mathrm{kHz}$ and $31.25 \mathrm{~ms}$ real time respectively. The sampling was again monitored by a plotter. Figure 1 shows the outline of our method of analysis.

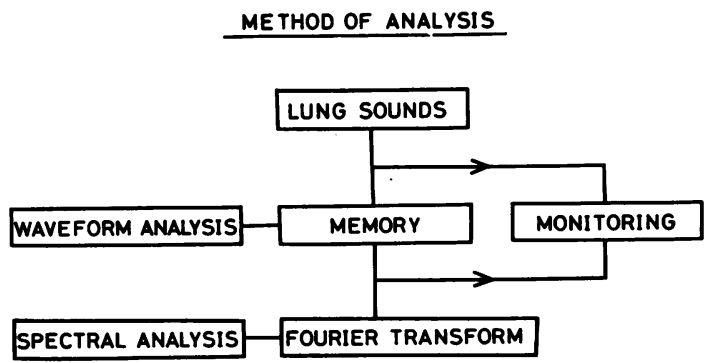

Fig 1 Block diagram of our method of analysis. For the waveform and spectral analysis of crackles monitoring at the time of sampling is necessary. 


\section{Results}

From six patients we sampled 245 crackles, of which 168 waveforms were selected for the measurement of half-periods. The rest were excluded because the tracings were either poor or overlapped by other crackles. By monitoring (fig 2) we could identify and sample crackles with
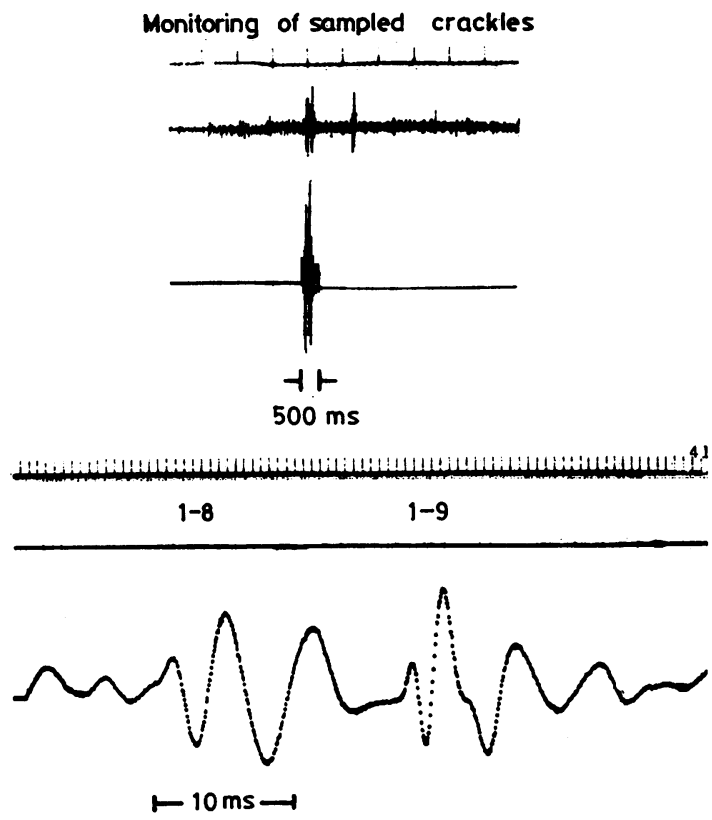

Fig 2 Example of monitoring (upper panel) and display of sampled waveforms (lower panel). We gave patient and serial numbers to each crackle. For instance, 1-8 represents number 8 crackle sampled from patient 1 .

almost identical waveforms in successive inspiratory or expiratory cycles (figs 3-7). The waveform of a crackle had between four and 10 positive and negative deflections. The interval of each deflection (half-period) was shortest $(0.5-2.0 \mathrm{~ms})$ initially and became progressively longer until a constant value $(2 \cdot 8-5 \cdot 2 \mathrm{~ms})$ was reached (fig 8 and table 1). In most waveforms we could identify an initial starting segment and subsequent decay segment (figs 3-7). This transition was more obvious in large amplitude waveforms than in low amplitude waveforms and in the starting segments of the former we observed steepenings of wavefronts and abrupt interruptions of waveforms by sudden deflections (figs 4-7). In 108 crackles $(44 \%)$ the decay segments lasted more than a cycle and appeared

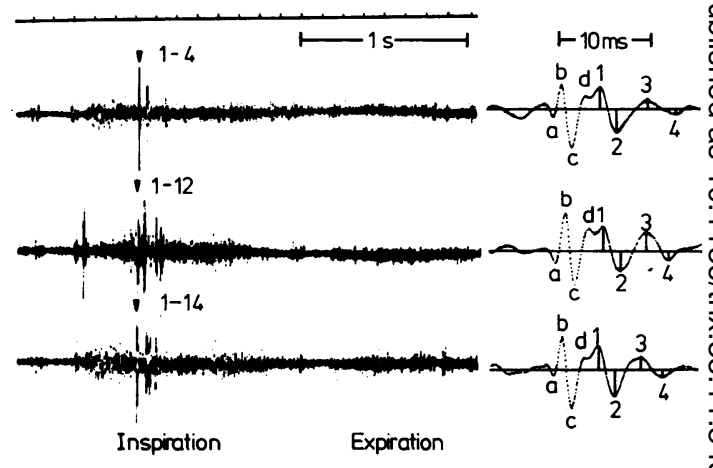

Fig 3 Left: amplitude versus time plots of lung sounds recorded from patient 1, pulmonary tuberculosis and thoracoplasty left. The site of recording was left base, posterior. Right: time expanded waveform analysis of crackles sampled from the same patient during three consecutive respiratory cycles. Each waveform on the right side corresponds to the crackle on the left side indicated by an arrow. Segments of waveform indicated by letters $(a, b, c, d)$ represent starting segments and those indicated by figures $(1,2,3,4)$ represent decay segments. Note repetitive appearance of crackles with almost identical waveforms.

damped sinusoids with almost exponential $\stackrel{2}{\circ}$ attenuations (figs $3,5,7,9$ ). The frequency of the decay segments ranged between $100 \mathrm{~Hz}$ to응 $300 \mathrm{~Hz}$ and within this low frequency range we observed no resonant response in our recording system.

Twelve representative crackles (two crackles for each patient) were selected for the spectral analysis. The spectrum of a crackle had $a_{0}^{\times}$ maximum peak at about $130 \mathrm{~Hz}$ to $220 \mathrm{~Hz}$ (fig $10 \exists$ and table 2) and the inverse of this frequency

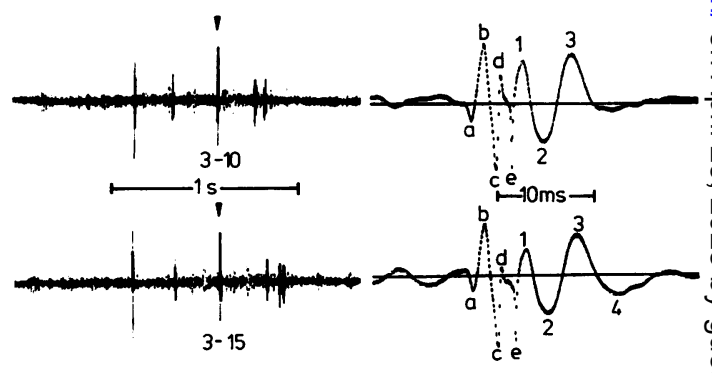

Fig 4 Left: amplitude versus time plots of lung sounds recorded from patient 3 , chronic bronchitis. The site of recording was right base, posterior.

Right: time expanded waveform analysis of crackles sampled during two consecutive inspiratory cycles. Labelling as fig 3. Note an abrupt interruption of waveforms by a sudden deflection (e). 


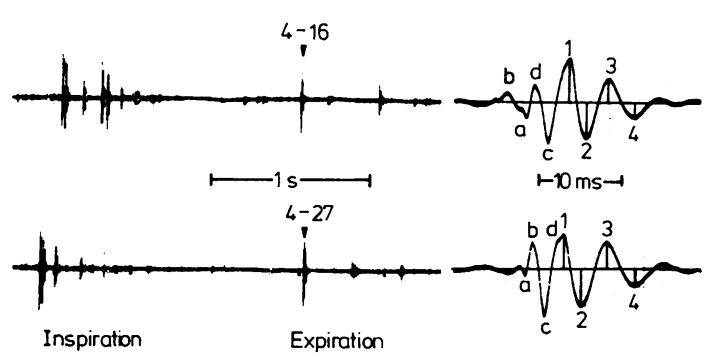

Fig 5 Left: amplitude versus time plots of lung sounds recorded from patient 4, pulmonary tuberculosis, right. The site of recording was right mid-lung field, posterior. Right: time expanded waveform analysis of crackles sampled during two consecutive expiratory cycles. Labelling as fig 3.

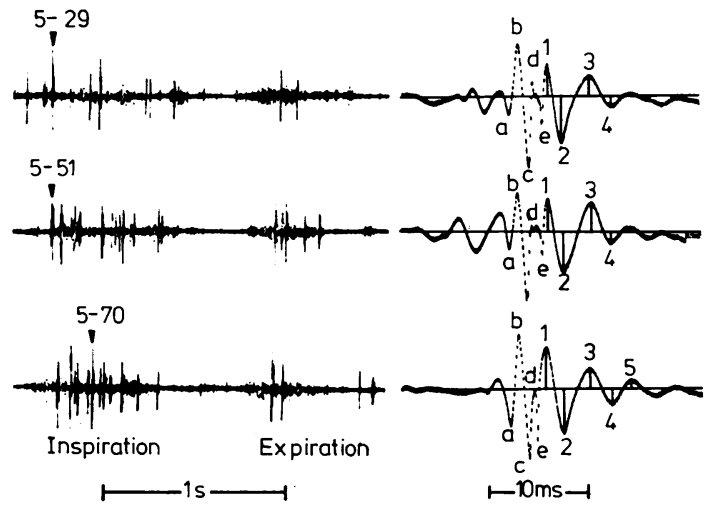

Fig 6 Left: amplitude versus time plots of lung sounds recorded from patient 5 , pulmonary tuberculosis, right. The site of recording was right upper lung field. Right: time expanded waveform analysis of early inspiratory crackles sampled during three consecutive respiratory cycles. Labelling as fig 3. Note abrupt deflections indicated by $d$ and $e$.
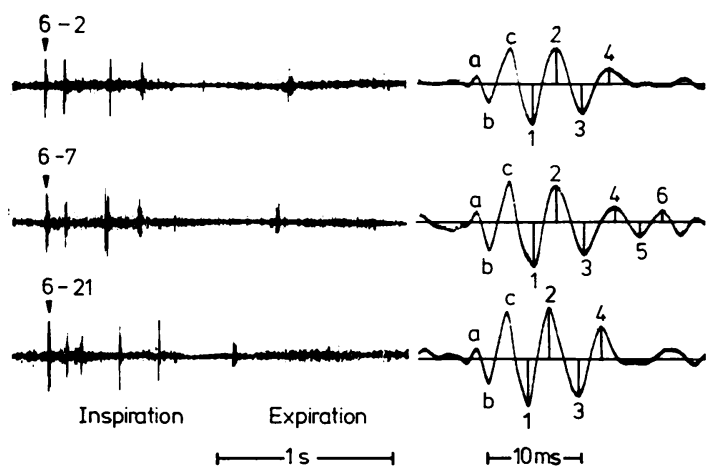

Fig 7 Left: amplitude versus time plots of lung sounds recorded from patient 6 , pulmonary tuberculosis, left. The site of recording was left upper lung field. Right: time expanded waveform analysis of early inspiratory crackles sampled during three consecutive respiratory cycles. Labelling as fig 3. Note steepening of wavefront at the junction of starting segment $(a, b, c)$ and decay segment $(1,2,3,4)$.

Table 2 Frequency " $F$ " obtained from the spectral analysis and intervals of decay segments obtained from the waveform analysis

\begin{tabular}{lllll}
\hline Patient & Crackle number & $F(\mathrm{~Hz})$ & $1 / F(\mathrm{~ms})$ & $T_{1-3}$ or $T_{2-4}(\mathrm{~ms})$ \\
\hline 1 & $1-2$ & $170 \cdot 7$ & $5 \cdot 86$ & $5 \cdot 58$ \\
& $1-14$ & $170 \cdot 7$ & $5 \cdot 86$ & $5 \cdot 56$ \\
\hline 2 & $2-34$ & 128 & $7 \cdot 81$ & $7 \cdot 13$ \\
& $2-43$ & 160 & $6 \cdot 25$ & $6 \cdot 35$ \\
\hline 3 & $3-10$ & 224 & $4 \cdot 46$ & $4 \cdot 93$ \\
& $3-15$ & 192 & $5 \cdot 21$ & $5 \cdot 24$ \\
\hline 4 & $4-16$ & 160 & $6 \cdot 25$ & $6 \cdot 00$ \\
& $4-27$ & 160 & $6 \cdot 25$ & $6 \cdot 27$ \\
\hline 5 & $5-29$ & 224 & $4 \cdot 46$ & $4 \cdot 28$ \\
& $5-51$ & 224 & $4 \cdot 46$ & $4 \cdot 47$ \\
\hline 6 & $6-2$ & 176 & $5 \cdot 68$ & $5 \cdot 31$ \\
& $6-7$ & 192 & $5 \cdot 21$ & $5 \cdot 43$ \\
\hline
\end{tabular}

Table 1 Clinical diagnosis and intervals of each deflection (half-periods) obtained from the waveform analysis

\begin{tabular}{|c|c|c|c|c|c|c|c|c|}
\hline \multirow{2}{*}{ Patient } & \multirow{2}{*}{ Diagnosis } & \multicolumn{7}{|c|}{ Half-period $(m s \pm S D)$} \\
\hline & & $a$ & $b$ & $c$ & $d(1)$ & 2 & 3 & 4 \\
\hline 1 & TB & $\begin{array}{l}1 \cdot 36 \pm 0 \cdot 54 \\
(14)\end{array}$ & $\begin{array}{l}1.91 \pm 0.58 \\
(14)\end{array}$ & $\begin{array}{l}2.35 \pm 0.65 \\
(14)\end{array}$ & $\begin{array}{l}3 \cdot 18 \pm 0 \cdot 36 \\
(14)\end{array}$ & $\begin{array}{l}2.98 \pm 0.65 \\
(14)\end{array}$ & $\begin{array}{l}3 \cdot 16 \pm 0 \cdot 23 \\
(14)\end{array}$ & - \\
\hline 2 & Chronic bronchitis & $\begin{array}{l}1.02 \pm 0 \cdot 29 \\
(40)\end{array}$ & $\begin{array}{l}1.47 \pm 0.40 \\
(40)\end{array}$ & $\begin{array}{l}1.94 \pm 0.46 \\
(40)\end{array}$ & $\begin{array}{l}2 \cdot 39 \pm 0.60 \\
(40)\end{array}$ & $\begin{array}{l}2.94 \pm 0.75 \\
(38)\end{array}$ & $\begin{array}{l}3 \cdot 37 \pm 0.79 \\
(27)\end{array}$ & $\begin{array}{l}3 \cdot 73 \pm 0.92 \\
(21)\end{array}$ \\
\hline 3 & Chronic bronchitis & $\begin{array}{l}0.93 \pm 0.37 \\
(18)\end{array}$ & $\begin{array}{l}1 \cdot 53 \pm 0 \cdot 27 \\
(18)\end{array}$ & $\begin{array}{l}2 \cdot 00 \pm 0 \cdot 51 \\
(15)\end{array}$ & $\begin{array}{l}2 \cdot 51 \pm 0.32 \\
(15)\end{array}$ & $\begin{array}{l}2.97 \pm 0.42 \\
(15)\end{array}$ & $\begin{array}{l}3 \cdot 22 \pm 0 \cdot 39 \\
(12)\end{array}$ & - \\
\hline 4 & TB & $\begin{array}{l}0 \cdot 72 \pm 0 \cdot 14 \\
(23)\end{array}$ & $\begin{array}{l}1 \cdot 41 \pm 0 \cdot 31 \\
(23)\end{array}$ & $\begin{array}{l}1 \cdot 86 \pm 0 \cdot 33 \\
(23)\end{array}$ & $\begin{array}{l}2 \cdot 72 \pm 0.54 \\
(23)\end{array}$ & $\begin{array}{l}3.09 \pm 0.51 \\
(18)\end{array}$ & $\begin{array}{l}3 \cdot 81 \pm 0 \cdot 77 \\
(15)\end{array}$ & $\begin{array}{l}4 \cdot 48 \pm 0 \cdot 71 \\
(14)\end{array}$ \\
\hline 5 & TB & $\begin{array}{l}1.04 \pm 0.47 \\
(46)\end{array}$ & $\begin{array}{l}1 \cdot 37 \pm 0 \cdot 59 \\
(46)\end{array}$ & $\begin{array}{l}1.69 \pm 0.57 \\
(44)\end{array}$ & $\begin{array}{l}2 \cdot 08 \pm 0.63 \\
(44)\end{array}$ & $\begin{array}{l}2 \cdot 58 \pm 0.64 \\
(32)\end{array}$ & $\begin{array}{l}2 \cdot 84 \pm 0 \cdot 71 \\
(14)\end{array}$ & $\begin{array}{l}2 \cdot 77 \pm 0 \cdot 61 \\
(6)\end{array}$ \\
\hline 6 & TB & $\begin{array}{l}1.46 \pm 0.49 \\
(27)\end{array}$ & $\begin{array}{l}1 \cdot 76 \pm 0 \cdot 62 \\
(27)\end{array}$ & $\begin{array}{l}2 \cdot 32 \pm 0.54 \\
(27)\end{array}$ & $\begin{array}{l}2 \cdot 62 \pm 0.39 \\
(27)\end{array}$ & $\begin{array}{l}3.09 \pm 0.36 \\
(25)\end{array}$ & $\begin{array}{l}3.02 \pm 0.46 \\
(20)\end{array}$ & $\begin{array}{l}3.46 \pm 0.42 \\
\text { (12) }\end{array}$ \\
\hline
\end{tabular}

Figures in parentheses represent numbers of crackles counted for each subject. 


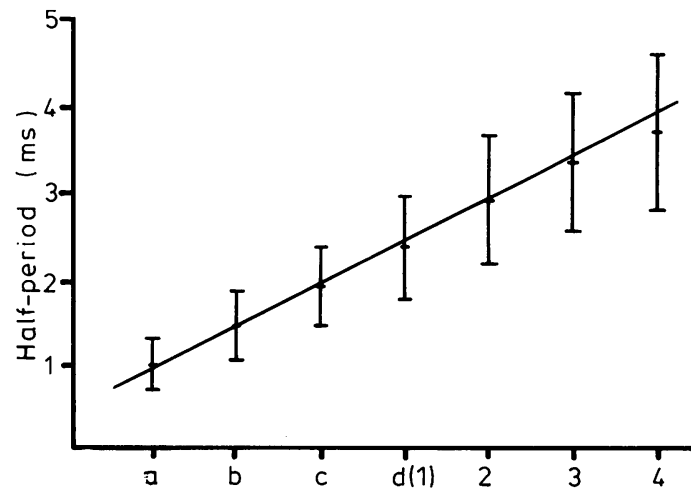

Fig 8 Half-periods, measured by zero-crossing method, of crackles obtained from patient 2. Note progressive elongation of half-periods with the development of waveforms. Vertical bars indicate mean $\pm S D$.

(F) was about equal to the period of decay segment $\left(T_{1-3}\right.$ or $\left.T_{2-4}\right)$, obtained from the waveform analysis (figs 10, 11). Spectral components beyond $1 \mathrm{kHz}$ were less than $2 \%$ of the maximum level (fig 10).

\section{Discussion}

Our method of time expansion for the waveform analysis is basically the same as previously employed by Murphy et $a l^{3}$ with the difference that we monitored each sampling to identify every crackle in a particular inspiratory or expiratory cycle.

In our waveform and spectral analysis the half-periods of a crackle ranged from $0.5 \mathrm{~ms}$ to $5 \mathrm{~ms}$ and in most crackles the spectral components beyond $1 \mathrm{kHz}$ were small (less than $2 \%$ of the maximum level). In our model experiment, in which crackles were simulated by exciting a resonator with shock waves (appendix), the halfperiods ranged from $1 \mathrm{~ms}$ to $3 \mathrm{~ms}$ and the spectral components beyond $1 \mathrm{kHz}$ were also less than $2 \%$ of the maximum level. Therefore we may assume that major frequency components of a crackle exist within a frequency range of $0.1 \mathrm{kHz}$ to $1 \mathrm{kHz}$ and that because of this limited frequency range the effect of chest wall interaction to modify transducer response is small.

For the spectral analysis, sampled waveforms should be correctly monitored because the result is different if, instead of a single crackle, more than two crackles are sampled in the same time window. ${ }^{4}$ For this reason spectral analysis of crackles by a sound spectrograph, ${ }^{5}$ in which sampled waveforms were not monitored, has $\frac{\bar{\sigma}}{\bar{s}}$ limitations.

By observing the time interval between con- $\propto$ secutive crackles and their relative loudness, $\infty$ Forgacs $^{1}$ has pointed out a recurrent pattern $\vec{\circ}$ in consecutive respiratory cycles as one of the most striking features of crackles. His obser- $\vec{\omega}$ vation was further supported by Nath and Capel ${ }^{2} \stackrel{\odot}{\rightleftharpoons}$ who reported that the timing of an individual $x$ crackle was closely associated with a particular transpulmonary pressure. Our observation of $\mathrm{a}-$ repetitive appearance of crackles with almostoo identical waveforms is further evidence in support of his observation.

Crackles are characterised by transient wave- forms of short duration with amplitudes greaterz than those of background breath sounds. ${ }^{3}$ In our?

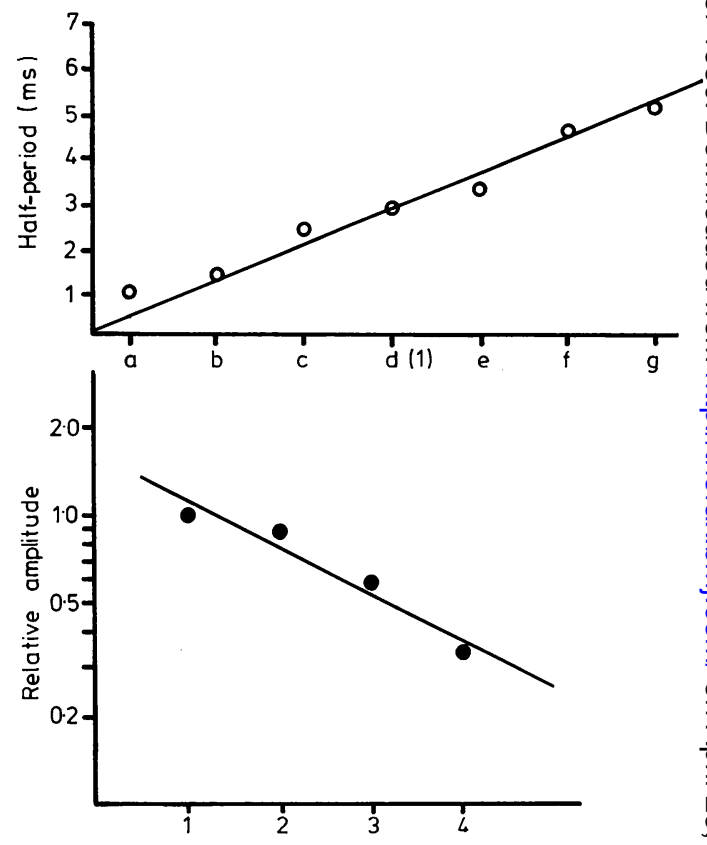

Fig 9 Upper panel: wave-heads, as indicated in fig 10, of a crackle (1I-43) on the abscissa and corresponding half-periods $(\mathrm{ms})$ on the ordinate.

The best fit line was calculated by least square method $(r=0.99)$. Note an increase of half-periods with the development of the waveform. Lower panel: wave-heads of the decay segment on the abscissa and the relative amplitudes on the ordinate. The decay of the amplitude is almost exponential. The best fit line was calculated by least square method $(r=-0.97)$. The quality factor $(Q)$ of the resonator $\mathbb{Q}$ determined from the slope (gradient) of the line was్ $4 \cdot 32$ (see Appendix). 


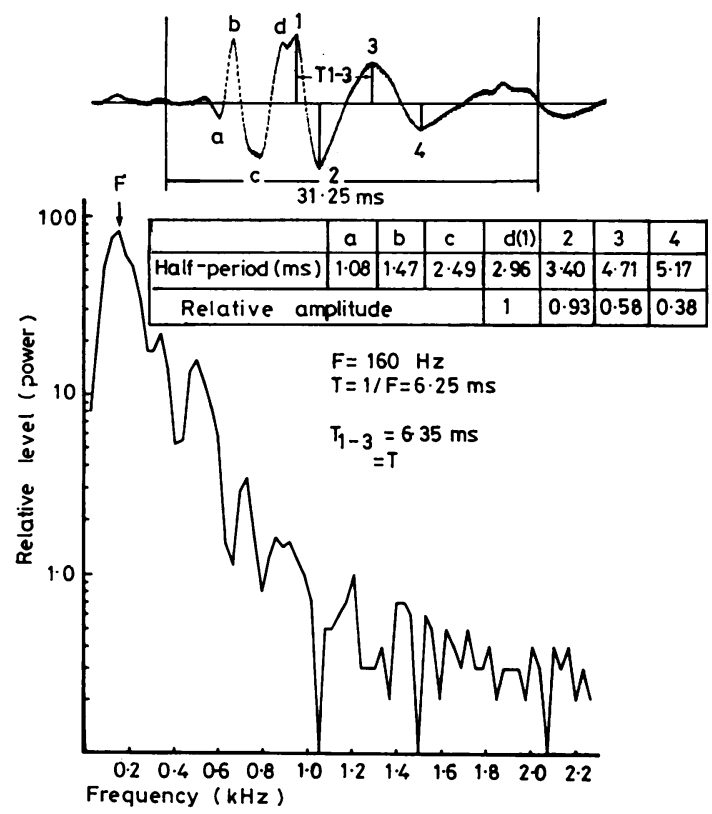

Fig $10 A$ representative waveform (upper panel) and power spectrum (lower panel) of a crackle (II-43) sampled from patient 2. The time window and sampling frequency were $31.25 \mathrm{~ms}$ and $64 \mathrm{kHz}$ real time respectively. $T_{1-3}$ is the time interval (ms) between "peak 1" and "peak 3" as indicated by arrows (upper panel). Half-periods were measured by zero-crossing method. $F$ is the frequency at which the power spectrum is maximum. In this crackle $F$ was $160 \mathrm{~Hz}$ and $1 / \mathrm{F}, 6 \cdot 25 \mathrm{~ms}$, which was about equal to $T_{1-3}(6.35 \mathrm{~ms})$.

waveform analysis we noticed several other characteristics. First, the intervals of each deflection (half-periods) were short initially and became progressively longer with the development of the waveforms. Second, most waveforms were composed of two segments, initial starting segments and subsequent decay segments. Third, in starting segments characteristics of shock waves such as steepenings of wave-fronts or discontinuities of waveforms ${ }^{6}$ were observed. Fourth, in fully developed waveforms the decay segments appeared damped sinusoids and the attenuations were exponential. Based on these observations we suggest that the initial starting segments represent shock waves caused by an abrupt opening of the airways, and that the subsequent decay segments are the result of these shock waves exciting a resonator in the lung. Progressive elongation of half-periods is also explainable on this basis because the wave travels momentarily at a speed greater than the undisturbed sound speed when a shock wave is produced.

Murphy et $a l^{3}$ observed that the tracings of fine crackles heard in patients with pulmonary fibrosis showed shorter periods than those of coarse crackles heard in patients with pneumonia. The causes of such difference have not been discussed. We speculate that the starting segment is determined primarily by the pressure ratio at the site of the airway opening, and this pressure ratio may increase with the increase of either the stiffness of the lung or the viscocity of mucus obliterating the airway. Therefore, one possible explanation is that in pulmonary fibrosis periods of initial segments are short because the lung is stiff and, as a result, the pressure ratio is high. The decay segment, on the other hand, is determined by the resonant frequency and the quality factor of the resonator. The waveforms recorded on the chest wall are modified further by attenuation and selective filtration during transmission and also by positional relationship between the sound source and the microphone, because, as pointed out by Forgacs, ${ }^{7}$ the pattern of crackles changes when the microphone or the stethoscope

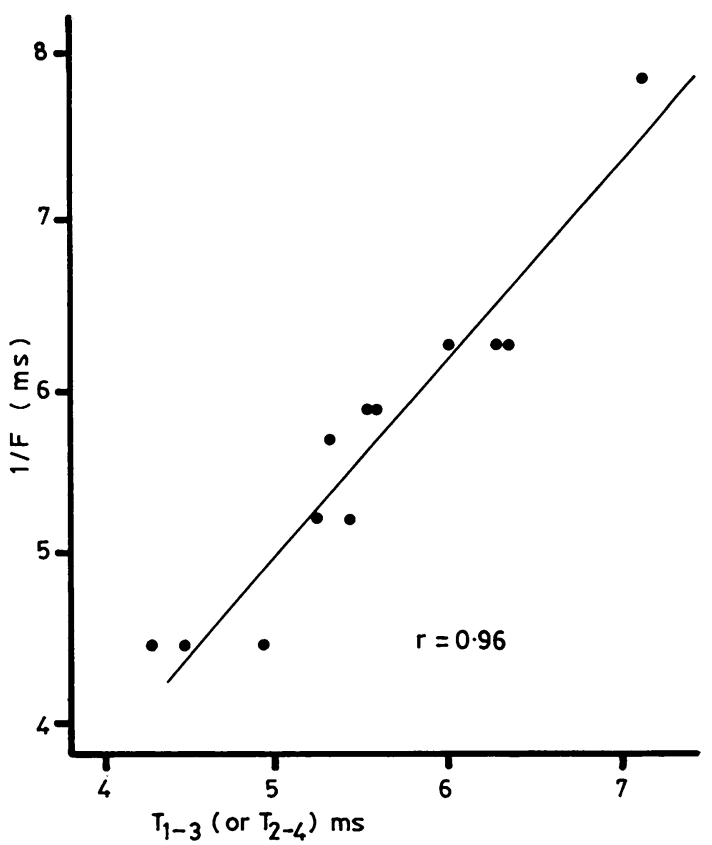

Fig 11 Correlation between $T_{1-3}\left(\right.$ or $\left.T_{2-4}\right)$ and $1 / F$ in 12 crackles (table 2). The former was obtained by waveform analysis and the latter by spectral analysis as indicated in fig 10. The best fit line was calculated by least square method. 
is moved over a short distance (fig 12). This highly directional transmission is another important characteristic of crackles. We observed fully developed waveforms in 108 out of 245 crackles, which indicates that crackles originated directly under the microphone were about $40 \%$ in our present study.

To test our hypothesis we devised a model experiment (described in the appendix) and were successful in obtaining waveforms similar to those of crackles. ${ }^{3}$
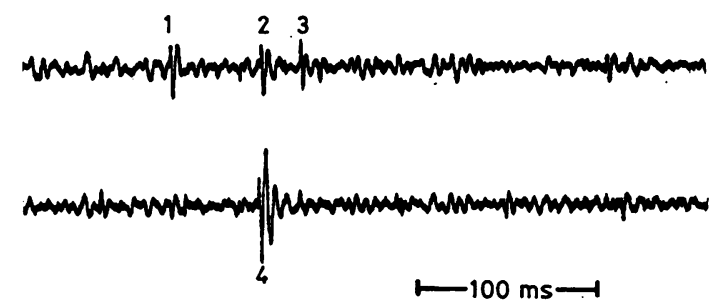

$6.25 \mathrm{~ms} /$ division
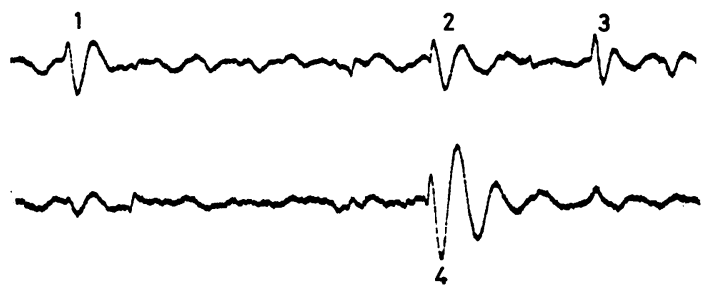

Fig $12 A$ simultaneous recording of inspiratory crackles at the right base from a patient with rheumatoid arthritis. For the recording two identical microphones (Sony ECM 150) were placed $4 \mathrm{~cm}$ apart horizontally. With the microphone fitted in the air chamber (volume: $2 \mathrm{~cm}^{3}$ ) the overall response of the recording system was flat within $\pm 2 d B$ in the range from 0.1 to $10 \mathrm{kHz}$. To avoid the effect of cavity resonance the internal surface of the air chamber and the contact surface were covered by sheets of polyurethane foam of $0.5 \mathrm{~cm}$ thickness. Note two waveforms in the upper panel (no 1 and no 3) and their poor counterparts in the lower panel. Though representing the same crackle, no 2 in the upper panel and no 4 in the lower panel have different waveforms. The decay segment is more distinct in the latter than in the former.

\section{References}

1 Forgacs P. Crackles and wheezes. Lancet 1967; 2:203-5.

2 Nath AR, Capel LH. Inspiratory crackles and mechanical events of breathing. Thorax 1974;
29:695-8.

3 Murphy RLH Jr, Holford SK, Knowler WC. $\frac{\bar{\sigma}}{\frac{\sigma}{2}}$ Visual lung-sound characterization by time- $\mathbb{\Phi}$

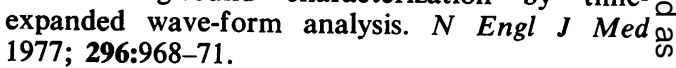

4 Yoganathan AP, Gupta R, Corcoran WH. Fast $\vec{\circ}$ Fourier transform in the analysis of biomedical data. Med Biol Eng Comput 1976; 14:239-45. $\vec{\omega}$

5 McKusick VA, Jenkins JT, Webb GN. The acoustic basis of the chest examination. Studies $x$ by means of sound spectrography. Am Rev W Tubercul Respir Dis 1955; 72:12-34.

6 Lighthill J. Shock waves. In: Waves in fluids. Cambridge: Cambridge University Press, 1978; $\frac{\infty}{\omega}$ 152-65.

7 Forgacs P. Crackles. In: Lung sounds. London: 옥 Baillière Tindall, 1978: 58.

\section{Appendix}

By waveform analysis we observed starting and decay segments in the waveforms of crackles. We speculated $\vec{P}$ that the former was a shock wave caused by an abrupto opening of the airway and that the latter was an. oscillation caused by the shock wave exciting aO resonator in the lung. To test our hypothesis we experimentally excited a resonator (a bottle) with a shock wave (fig 13). The quality factor of theo resonator $(Q)$ was reduced to about 5 by covering the $\frac{}{\infty}$ internal surface of the bottle with a sheet of $\varrho$ polyurethane foams of $0.9 \mathrm{~cm}$ thickness. The sound $\overrightarrow{\vec{O}}$ was recorded and analysed in the same way as $\exists$ previously described for the crackles. Figure 14 shows a representative waveform and the result of spectralo analysis. As in the waveform of crackles initial starting segment and subsequent decay segment were observed. Elongation of half-periods and exponentialo decay of amplitudes with the development of the $e_{-}^{x}$ waveform were also observed (fig 15). The frequency 3 $\mathrm{F}$, at which the spectrum became maximum, and the frequency of the decay segment were about equal and agreed with the resonant frequency of the bottle (with a sheet of polyurethane foams inside) used as a resonator (fig 14).

Assuming that our model is equivalent to a seriesㅇㅡㅡ RLC electrical circuit, the decay segment can be approximated to a damped sinusoid with its envelopeos given by

$$
\mathrm{V}=\mathrm{Vo} \cdot \mathrm{e}^{-\alpha \mathrm{t}} \text {. }
$$

where $\mathrm{V}$ is the relative amplitude for each wave $\mathrm{C}$ head, Vo is the initial amplitude, $\alpha$ is the rate of attenuation, and $t$ is time. The quality factoro (Q) of this circuit is,

$$
Q=2 \pi f \cdot L / R,
$$

where $f$ is the resonant frequency, $L$ and $R$ are the inductance and the resistance respectively. Since $\alpha=R / 2 L$,

$$
\mathrm{L} / \mathrm{R}=1 / 2 \alpha
$$

By substituting equation 2 into equation $1, \stackrel{0}{2}$ becomes,

$$
\mathrm{Q}=\pi \mathrm{f} / \boldsymbol{\alpha},
$$




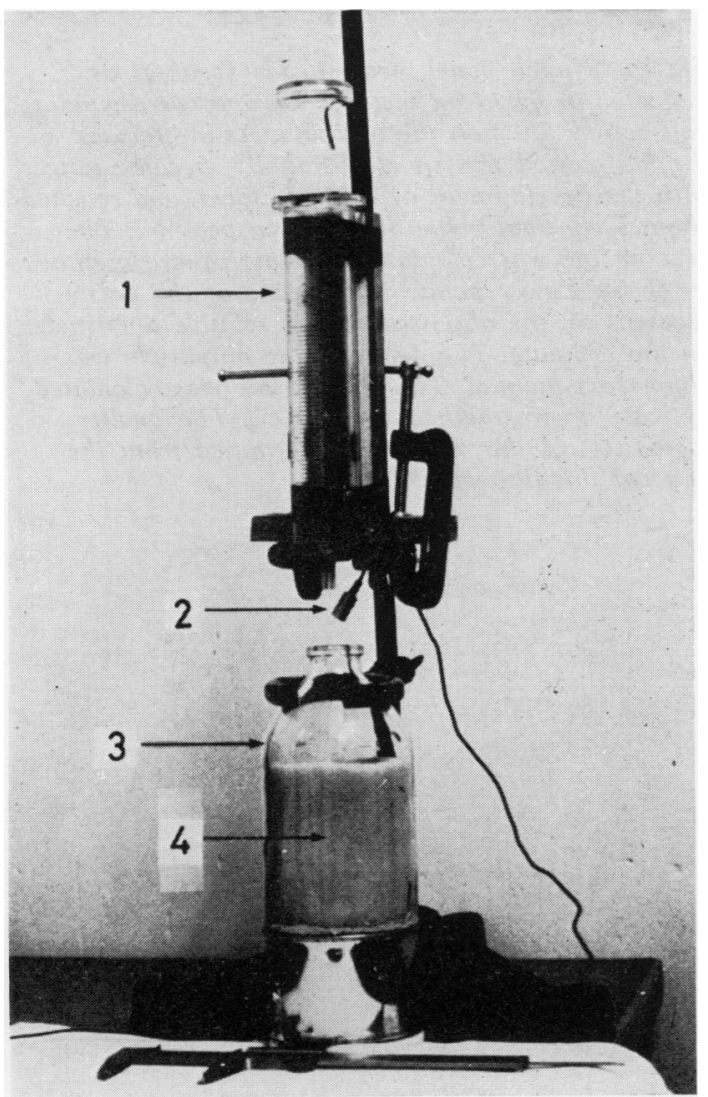

Fig 13 We simulated crackles by this model experiment. A syringe (I) and a microphone (Sony, ECM 150) (2) were positioned above an emptied bottle (3) which was used as a resonator. The quality factor of the resonator $(Q)$ was reduced to about 5 by covering the internal surface of the bottle with a sheet of polyurethane foam of $0.9 \mathrm{~cm}$ thickness (4). The resonator was excited by a weak shock wave which was generated by applying commercial paste (Arabic Yamato, dynamic viscosity: 3-4 SI) at the orifice of the syringe and moving the piston up and down slowly. The vernier caliper at the bottom indicates $5 \mathrm{~cm}$.

where $f$ can be measured and $\alpha$ can be calculated from the rate of decay (gradient) of the relative amplitudes (fig 15 , lower panel). $Q$ determined from equation 3 in this model experiment was 5.9 , which agreed well with the quality factor (Q) of 4.9 determined separately from the equation.

$$
Q=f / \Delta f
$$

where $\Delta f$ is the bandwidth off resonance at which the response is 0.707 of the maximum (resonant) response.

We calculated $Q$ in our patients from equation 3 and found the equivalent $Q$ of the lung around the frequency range of 150 to $250 \mathrm{~Hz}$ was about 3 to 6 . An example was given in fig 10 . Equation 3 can be simplified further as follows,

$$
\begin{aligned}
\alpha & =2 \mathrm{k} \cdot 1 / \mathrm{T} \cdot 1 \mathrm{n} 10 \\
& =2 \mathrm{k} \cdot \mathrm{f} \cdot \ln 10
\end{aligned}
$$

where $T$ and $f$ are the period and the frequency of the decay segment respectively $(T=6.35 \mathrm{~ms}$, $f=1 / T=157 \mathrm{~Hz}$, fig 9), and $k$ is the gradient (per half-period, fig 10, lower panel). Multiplication by 1 in 10 is necessary for the conversion of the base of a logarithm (from a common logarithm to a natural logarithm). By substituting equation 5 into equation 3 , equation 3 becomes,

$$
\begin{aligned}
\mathrm{Q} & =\pi / 2 \mathrm{k} \cdot \ln 10 \\
& =0 \cdot 682 / \mathrm{k}
\end{aligned}
$$

In fig 10 (lower panel) $\mathrm{k}=-0.158, \mathrm{Q}=0.682 / 0 \cdot 158$ $=4 \cdot 32$.

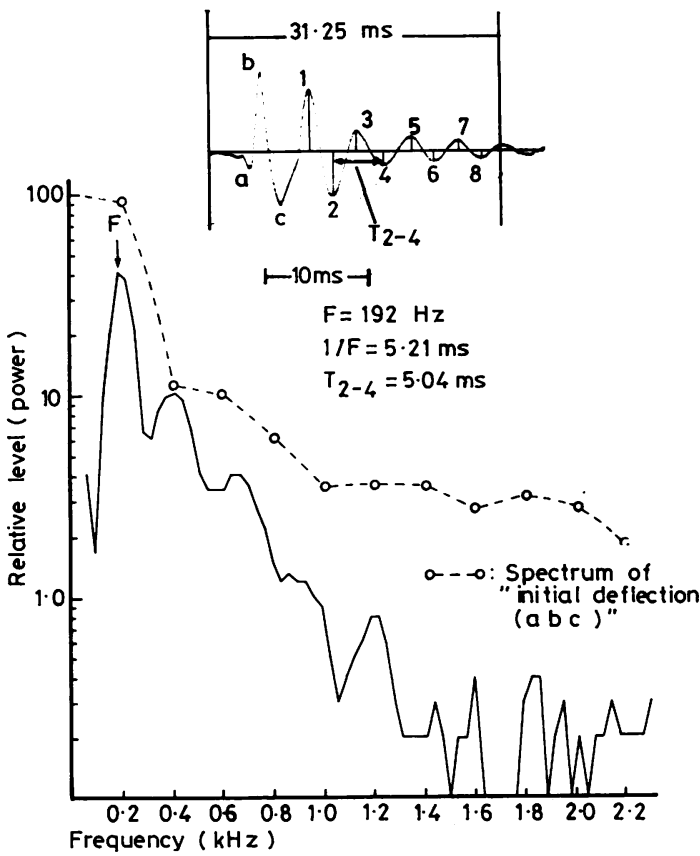

Fig $14 A$ representative waveform (upper panel) and the power spectrum (lower panel) of a simulated crackle. As in the waveforms of crackles starting and decay segments are indicated by letters and figures respectively. The resonant frequency of the resonator $(204 \mathrm{~Hz})$, the frequency of the decay segment $\left(1 / T_{2-4}=1000 / 5 \cdot 04=198 \mathrm{~Hz}\right)$, and the frequency $F$ at which the power spectrum became maximum, were all about equal. Spectral components beyond $1 \mathrm{kHz}$ are less than $2 \%$ of the maximum level. The spectrum (dotted lines) of the starting segment, which excited the resonator, also declines rapidly beyond $200 \mathrm{~Hz}$ and becomes the level of 3 to $4 \%$ of the maximum beyond $1 \mathrm{kHz}$. 


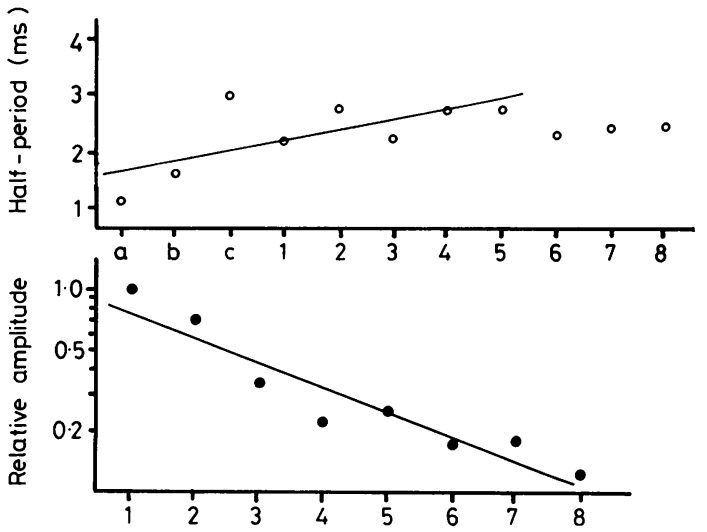

Fig 15 Upper panel: wave-heads (peaks), as indicated in fig 14 on the abscissa and corresponding half-periods (ms) on the ordinate. As in crackles the half-period was shortest initially, became longer with the development of the waveform, and reached about a constant value of $2.5 \mathrm{~ms}$ at peak 5". The best fit line was calculated by least square method $(r=0.68)$. Lower panel: wave-heads of the decay segment on the abscissa and the relative amplitudes on the ordinate. The decay of the amplitude was almost exponential. The best fit line was calculated by least square method $(r=-0.95)$. The quality factor $(Q)$ of the resonator determined from the slope of this line was 5.9. 